\title{
Prosthetic Treatment of Severely Resorbed Anterior Ridge: Case Report
}

\author{
Dr. K.Varun Reddy ${ }^{1}$, Dr. V.H.Chalapathi Kumar ${ }^{2}$, Dr. Pavan Kumar ${ }^{3}$, \\ Dr. P.Mahesh ${ }^{4}$ \\ ${ }^{I}$ (Postgraduate, Department of prosthodontics, Narayana dental college, Nellore, India) \\ ${ }^{2}$ (Professor, Department of prosthodontics, Narayana dental college, Nellore, India) \\ ${ }^{3}$ (Professor, Department of prosthodontics, Narayana dental college, Nellore, India) \\ ${ }^{4}$ (HOD/Professor, Department of prosthodontics, Narayana dental college, Nellore, India)
}

\begin{abstract}
Restoration of anterior ridge defects with missing teeth both in height and width is critical for a dentist to restore. Restoration of lost teeth along with the bony defect restricts many modes of conventional treatments available. For obtaining functionally and aesthetically acceptable results a combination of fixed and removable prosthesis has turned out to be outstanding without the need for rehabilitation of the defect with soft tissue and hard tissue defects. This article represents a case report of maxillary anterior ridge defect of Siebert's class III and management with Andrew's Bridge.
\end{abstract}

Keywords: Anterior Defect, Andrew's Bridge, Prosthesis, Siebert's classification.

\section{Introduction}

Prosthetic rehabilitation of the bone and soft tissue defects with restoration of function, esthetics and maintenance is often challenging for a prosthodontist. Anterior ridge deformities that often end up with loss of bone due to congenital defects, trauma, periodontal problems, periapical pathologies and traumatic extractions are contraindicated for restoration with conventional methods like removable partial denture, fixed partial denture [1,2]. Restoration of lost teeth with conventional fixed partial dentures resulted in increase in the height of the crowns, which are unaesthetic. Surgical recontouring of the defect with bone grafts and soft tissue augmentation is often time taking and cumbersome with a comprised prognostic situation. Treatment of such cases have been attempted since 1900's and turned out to be successful with the introduction of Andrew's Bridge by Dr. James A. Andrews, Covington, LA. Andrew's Bridge is a fixed removable partial denture which has a removable denture replacing the defect area and which rests on fixed bar attachment, which is attached to the retainers.

\section{Indications $[3,4,5]$}

1. Ridge defects which contraindicate the placement of conventional fixed partial denture.

2. Augmentation of the defect (grafting) area is contradicted due to systemic conditions of the patient.

3. Patients whose residual ridge has a relationship to the opposing dentition that would prohibit the esthetic placement of the pontics of a fixed partial denture.

4. Cleft palate and cleft lip defects.

\section{Case Report}

A 23 year old male patient named Tirumala, reported to the department of prosthodontics, Narayana dental college with a chief complaint of missing teeth in the upper front region. Patient had a history of cystic enucleation done 2 years back that resulted in loss of 21, 22, and 23. Patient did not present any significant medical history. On intraoral examination the residual alveolar ridge in the area of missing teeth found to be of Siebert's Class III [6]. Patient was explained with all the treatment options that are viable with the situation. Although implant placement was planned, but due to the need for preprosthetic surgeries like bone grafting and long term healing period with an unpredictable outcome, this option was eliminated. The patient was not satisfied with the removable denture fabricated and conventional fixed prosthesis was also not appropriate. So a fixed removable prosthesis (Andrew's Bridge) was planned for this situation.

\section{Procedure:}

1. Diagnostic impressions were made and casts were obtained with dental stone[fig 1,2]

2. Facebow transfer was done and the models were articulated for diagnostic wax up to assess the esthetics and for patient education. 
3. Tooth preparation of the abutment teeth were done $(13,12,11,24$, and 25$)$ to receive porcelain fused to metal crowns and impressions were made with polyvinyl siloxane impression material and master casts were poured with type IV gypsum [fig 3, 4].

4. Provisionalisation was done to the abutment teeth with tooth colour heat cure acrylic resin.

5. Wax patterns for the metal copings of the abutment teeth were made and 11 and 24 abutments were attached with prefabricated plastic bar attachment a $3 \mathrm{~mm}$ of gap was left between the plastic bar attachment and the crest of the ridge [fig 5].

6. Casting of the assembly was done.

7. The metal framework assembly was tried in the patient's mouth and ensured for proper marginal fit and clearance for ceramic. Shade selection was done for ceramic build up [fig 6].

8. With the metal framework in place, a pick up impression was made with polyvinyl siloxane impression material by single stage technique and cast was obtained for fabrication of denture.

9. Teeth arrangement was done of the selected shade and wax up was done with the clip attachment secured on the bar in the metal framework. Denture was processed with heat cure acrylic resin.

10. After finishing and polishing was done, proper seating and retention of the clip over the bar attachment was rechecked [fig 7, 8, 9]

11. Patient was instructed with the oral hygiene measures that were to be followed and follow up was done after 1 week [fig 10].

III. FIGURES

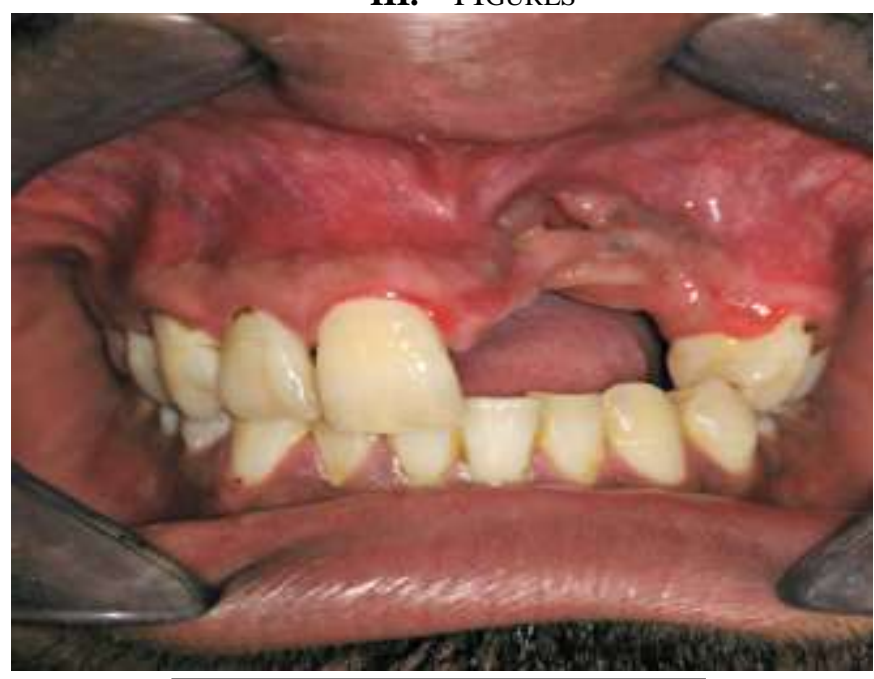

Fig 1. Preoperative Intraoral

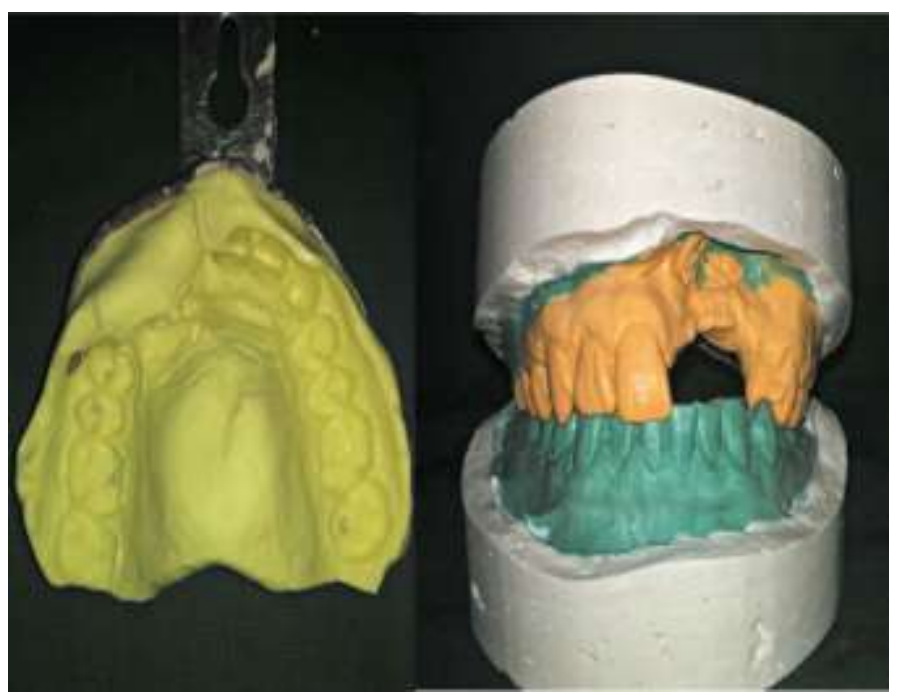

Fig 2. Primary impression and primary models 


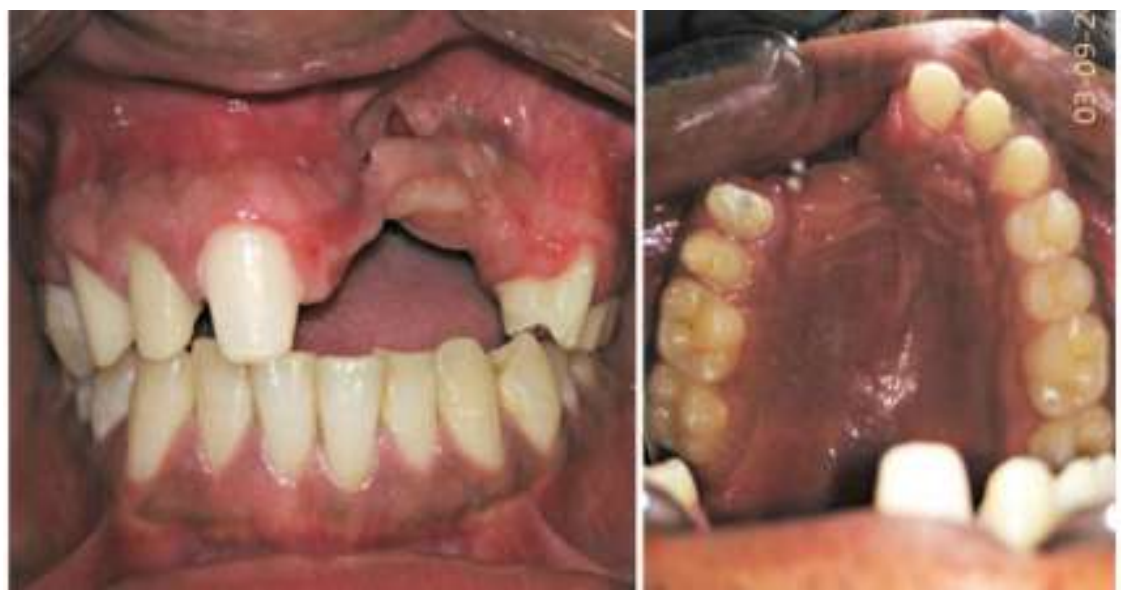

Fig 3. Tooth preparation of $13,12,11,24 \& 25$



Fig 4. Secondary impression

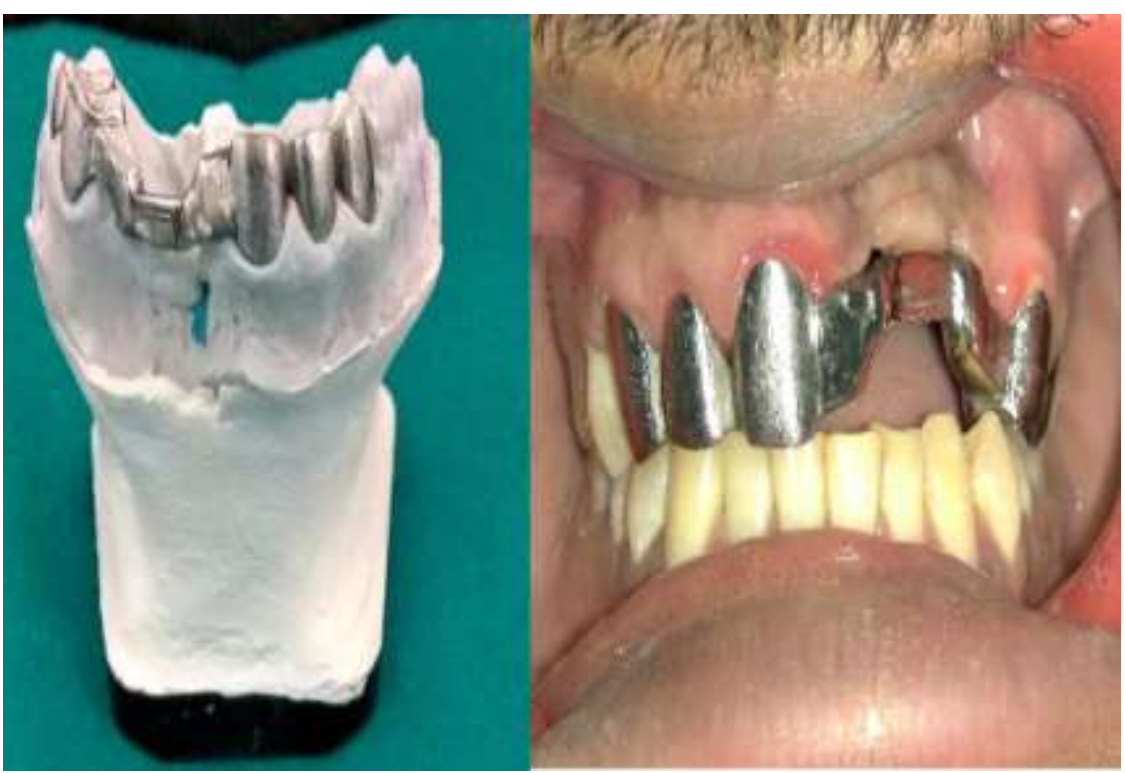

Fig 6. Metal framework and metal try in 


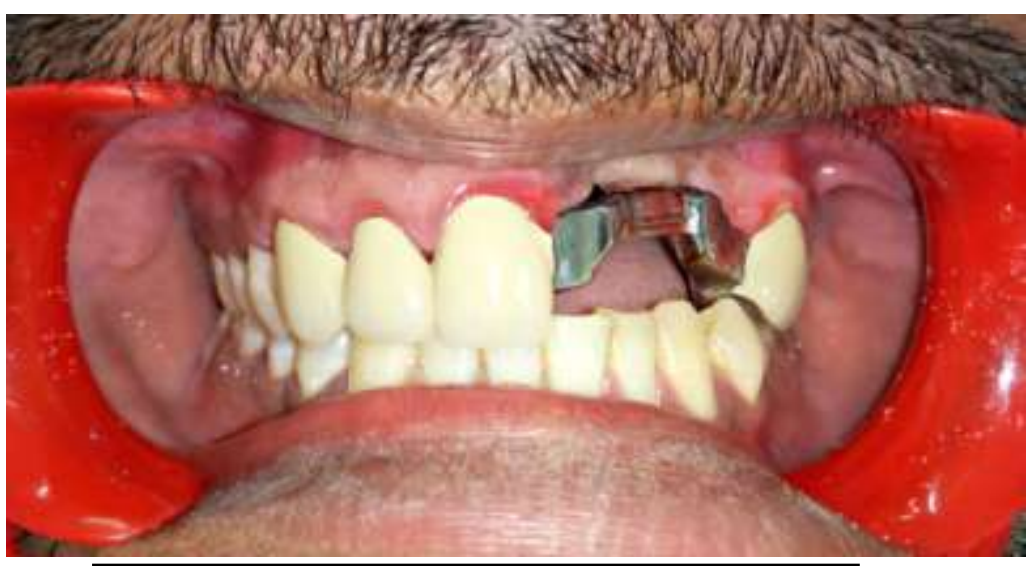

Fig 7. Cemented PFM crowns with bar attachment

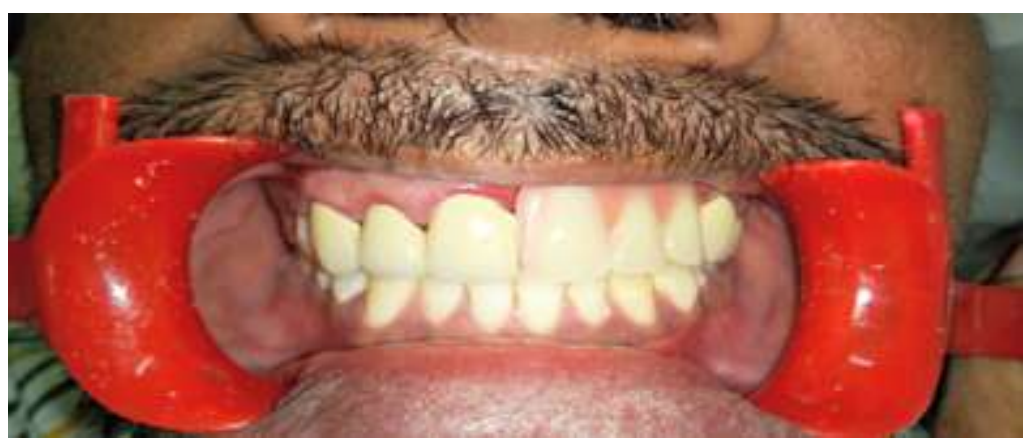

Fig 8. Postoperative intraoral view of Andrew's bridge

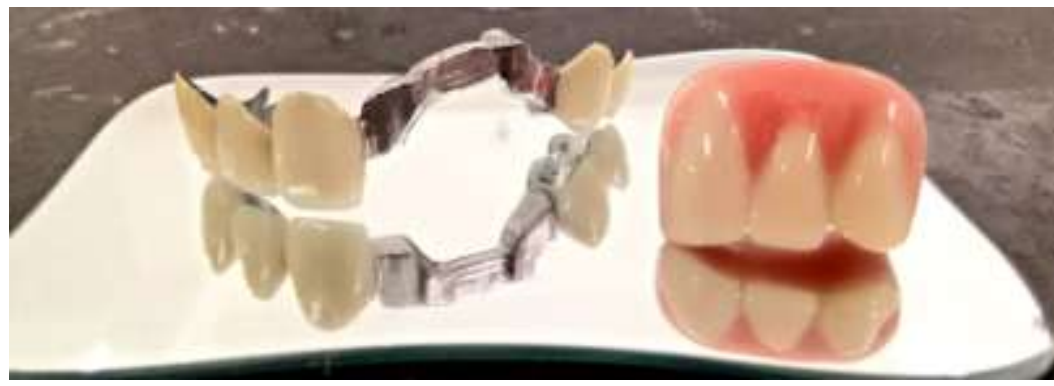

Fig 9. Fixed and removable components of Andrew's

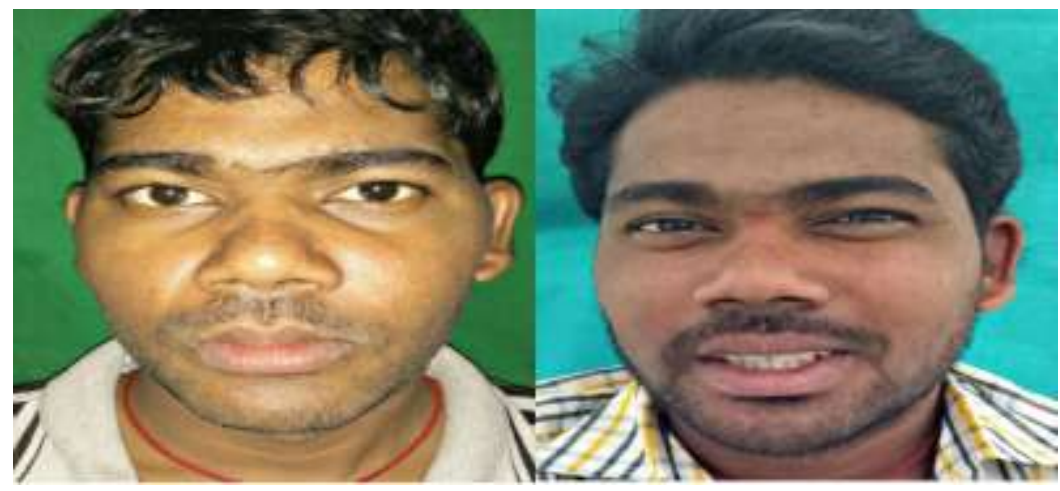

Fig 10. Preoperative and postoperative view 


\section{Discussion}

Missing teeth with the associated ridge defects has been classified by siebert [6] and the incidence of the occurrence of these defects has been documented by hebert abrams [7], which concluded the incidence of the anterior defects has exceptionally high incidence of $91 \%$. The resulting defect due to trauma or due to congenital causes may show variation in form, size and severity. The defect thus has to be examined carefully before planning or attempting to plan for the restoration [8]. Andrews bridge is an acceptable mode of treatment modality for the patient with such type of defects which reformates the function and esthetics. The severity of the defect is greater in case of siebert class iii situation as seen in this case report [fig. 1], with loss of width and height of the ridge. The fixed removable prosthesis offers advantages of both the removable and fixed prosthesis. The removable pontic denture helps in maintaining the esthetics by providing ample fullness whereas the fixed prosthesis retains the removable part with an attachment that showed to have an increase in retention and fit for the removable PROSTHESIS.

\section{Conclusion}

Andrew's Bridge is particularly indicated in patients where there is an extensive loss of supporting structure and when the esthetic arch position often possess difficulties. Selection of patient is critical and the problems occur essentially due to the diagnostic errors during the treatment planning. This fixed removable prosthesis is economical, hygienic and is comfortable to use [9].

\section{References}

[1]. Studer S, Naef R, Scharer P. Adjustment of localized alveolar ridge defects by soft tissue transplantation improves mucogingival esthetics: A proposal for clinical classification and evaluation of procedures. Quintessence Int. 1997;28(12):785- 805

[2]. Shankar, et al. A fixed removable partial denture treatment for severe ridge defect - A case report. Int J Dent Case Reports. 2011;1(2): 112-18.

[3]. Stein RS. Pontic - residual ridge relationship: A Research Report. J Prosthet Dent 1966;16(2):251-85.

[4]. Henderson, D., and Steffel, V. L.: McCracken's Removable Partial Prosthodontics. St. Louis, 1977, The C. V. Mosby Co., P 1.

[5]. Mueninghoff KA, Johnson MH. Fixed-removable partial denture. J Prosthet Dent 1982;48:547-50.

[6]. Seibert JS. Reconstruction of deformed, partially edentulous ridges, using full thickness onlay grafts. Part I. Technique and wound healing. Compend Contin Educ Dent. 1983;4(5):437-53

[7]. Abrams H, Kopczyk RA, Kaplan AL. Incidence of anterior ridge deformities in partial edentulous patients. J Prosthet Dent. 1987;57(2):191-94.

[8]. Everhart RJ, Cavazos E. Evaluation of a fixed removable partial denture: Andrews Bridge System. The Journal of prosthetic dentistry. 1983 Aug 1;50(2):180-4.

[9]. Sadig W.M. Bone Anchored Andrew's Bar System-A Prosthetic Alternative. Cairo Dental Journal. 1995;11:11-15. 